\title{
Structural Basis of EGFR Mutant Inhibition by Trisubstituted Imidazole Inhibitors
}

David E. Heppner, ${ }^{a, b}$ Marcel Günther, ${ }^{c}$ Florian Wittlinger, ${ }^{c}$ Stefan Laufer, ${ }^{c}$ Michael J. Eck ${ }^{a, b}$

aDepartment of Cancer Biology, Dana-Farber Cancer Institute, Boston, MA 02215, USA.

${ }^{b}$ Department of Biological Chemistry and Molecular Pharmacology, Harvard Medical School, Boston, MA 02115, USA.

'Institute for Pharmaceutical Sciences, Eberhard Karls Universität Tübingen, Tübingen 72076, Germany.

\section{Table of Contents}

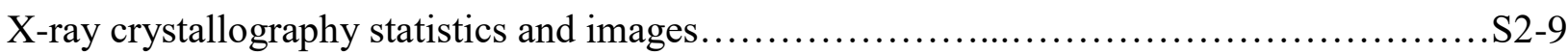

Biochemical Potencies of structurally characterized inhibitors................................. 10

Synthesis of $N$-(3-Bromo-4-methoxyphenyl)acrylamide .................................... 11 
Table S1. Crystallographic data collection and refinement statistics for EGFR(T790M/V948R) crystals.

\begin{tabular}{|c|c|c|c|}
\hline $\begin{array}{l}\text { PDB ID Code } \\
\text { Inhibitor }\end{array}$ & $\begin{array}{c}\text { 6V5N } \\
1 \text { (LN2084) }\end{array}$ & $\begin{array}{c}\text { 6V5P } \\
2(\mathrm{LN2725})\end{array}$ & $\begin{array}{c}\text { 6V6O } \\
3 \text { (LN2380) }\end{array}$ \\
\hline $\begin{array}{l}\text { Data collection } \\
\text { Space group }\end{array}$ & $\mathrm{P} 12_{1} 1$ & $\mathrm{P} 12_{1} 1$ & $\mathrm{P} 12_{1} 1$ \\
\hline $\begin{array}{l}\text { Cell dimensions } \\
\text { a, b, c }(\AA)\end{array}$ & $\begin{array}{c}71.1 \\
102.12 \\
87.38\end{array}$ & $\begin{array}{c}70.36 \\
101.61 \\
87.17\end{array}$ & $\begin{array}{c}71.5501 \\
102.36 \\
173.569\end{array}$ \\
\hline$\alpha, \beta, \gamma\left(^{\circ}\right)$ & $\begin{array}{c}90.0 \\
102.55 \\
90.0\end{array}$ & $\begin{array}{c}90.0 \\
102.31 \\
90.0\end{array}$ & $\begin{array}{c}90 \\
101.298 \\
90\end{array}$ \\
\hline Resolution* $(\AA)$ & 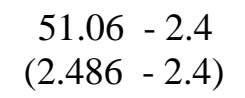 & $\begin{array}{c}40.24-2.3 \\
(2.382-2.3)\end{array}$ & $\begin{array}{c}60.81-2.1 \\
(2.175-2.1)\end{array}$ \\
\hline $\mathrm{R}_{\text {merge }} *$ & $\begin{array}{c}0.1217 \\
(0.7778)\end{array}$ & $\begin{array}{l}0.07567 \\
(0.6614)\end{array}$ & $\begin{array}{c}0.1149 \\
(0.5684)\end{array}$ \\
\hline $\mathrm{I} / \sigma^{*}$ & $12.72(2.10)$ & $13.91(2.44)$ & $11.04(4.04)$ \\
\hline Completeness* $(\%)$ & $98.71(97.35)$ & $99.11(98.87)$ & 98.45 (97.77) \\
\hline Multiplicity* & $6.9(5.9)$ & $5.2(5.0)$ & $7.0(7.0)$ \\
\hline $\begin{array}{c}\text { Refinement } \\
\text { Resolution }(\AA)\end{array}$ & $51.06-2.4$ & $40.24-2.3$ & $60.81-2.1$ \\
\hline No. of Reflections & 47126 & 52834 & 140813 \\
\hline $\begin{array}{c}\mathrm{R}_{\mathrm{work}} / \mathrm{R}_{\text {free }} \\
\text { No. of Atoms }\end{array}$ & $0.1921 / 0.2440$ & $0.1791 / 0.1937$ & $0.1945 / 0.2214$ \\
\hline Protein & 9779 & 9747 & 19612 \\
\hline Ligands/ion & 128 & 131 & 296 \\
\hline $\begin{array}{c}\text { Water } \\
\text { B-factors }\end{array}$ & 255 & 375 & 1618 \\
\hline Protein & 46.51 & 45.39 & 31.06 \\
\hline $\begin{array}{l}\text { Ligand/ion } \\
\text { Water }\end{array}$ & $\begin{array}{l}43.98 \\
44.67\end{array}$ & $\begin{array}{l}40.20 \\
45.97\end{array}$ & $\begin{array}{l}24.79 \\
36.76\end{array}$ \\
\hline R.m.s deviations & & & \\
\hline Bond lengths $(\AA)$ & 0.011 & 0.008 & 0.003 \\
\hline $\begin{array}{l}\text { Bond angles }\left({ }^{\circ}\right) \\
\text { Ramachandran }\end{array}$ & 1.14 & 0.91 & 0.68 \\
\hline Most favored & 94.92 & 96.01 & 97.33 \\
\hline Allowed & 4.00 & 3.41 & 2.42 \\
\hline Outliers & 1.08 & 0.50 & 0.25 \\
\hline
\end{tabular}


Table S1 continued.

\begin{tabular}{|c|c|c|}
\hline $\begin{array}{l}\text { PDB ID Code } \\
\text { Inhibitor }\end{array}$ & $\begin{array}{c}\text { 6V6K } \\
4 \text { (LN2057) }\end{array}$ & $\begin{array}{c}\text { 6V66 } \\
5 \text { (LN2899) }\end{array}$ \\
\hline $\begin{array}{l}\text { Data collection } \\
\text { Space group }\end{array}$ & $\mathrm{P} 12,1$ & $\mathrm{P} 12_{1} 1$ \\
\hline $\begin{array}{l}\text { Cell dimensions } \\
\text { a, b, c }(\AA)\end{array}$ & $\begin{array}{l}71.5988 \\
102.455 \\
174.043\end{array}$ & $\begin{array}{l}71.4802 \\
102.448 \\
87.4219\end{array}$ \\
\hline$\alpha, \beta, \gamma\left(^{\circ}\right)$ & 90101.2590 & 90102.77590 \\
\hline Resolution* $(\AA)$ & $\begin{array}{c}85.35-2.2 \\
(2.279-2.2)\end{array}$ & $\begin{array}{r}85.26-1.79 \\
(1.854-1.79)\end{array}$ \\
\hline $\mathrm{R}_{\text {merge }} *$ & $\begin{array}{l}0.08136 \\
(0.5284)\end{array}$ & $0.1081(0.796)$ \\
\hline $\mathrm{I} / \sigma^{*}$ & $7.80(2.11)$ & $9.59(1.95)$ \\
\hline Completeness* (\%) & $93.67(95.49)$ & $99.61(99.45)$ \\
\hline Multiplicity* & $3.1(3.1)$ & $7.0(7.1)$ \\
\hline $\begin{array}{c}\text { Refinement } \\
\text { Resolution }(\AA)\end{array}$ & $85.35-2.2$ & $85.26-1.79$ \\
\hline No. of Reflections & 369052 & 115036 \\
\hline $\begin{array}{c}\mathrm{R}_{\text {work }} / \mathrm{R}_{\text {free }} \\
\text { No. of Atoms }\end{array}$ & $0.2130 / 0.2394$ & $0.1844 / 0.2235$ \\
\hline Protein & 19759 & 9901 \\
\hline Ligands/ion & 280 & 160 \\
\hline $\begin{array}{c}\text { Water } \\
\text { B-factors }\end{array}$ & 712 & 996 \\
\hline Protein & 36.59 & 27.53 \\
\hline Ligand/ion & 29.34 & 23.88 \\
\hline Water & 38.02 & 34.75 \\
\hline $\begin{array}{l}\text { R.m.s deviations } \\
\text { Bond lengths }(\AA)\end{array}$ & 0.007 & 0.007 \\
\hline $\begin{array}{l}\text { Bond angles }\left(^{\circ}\right) \\
\text { Ramachandran }\end{array}$ & 0.95 & 0.85 \\
\hline Most favored & 96.66 & 97.62 \\
\hline Allowed & 3.01 & 2.14 \\
\hline Outliers & 0.33 & 0.25 \\
\hline
\end{tabular}

*Numbers in parentheses are for the highest resolution shell. 

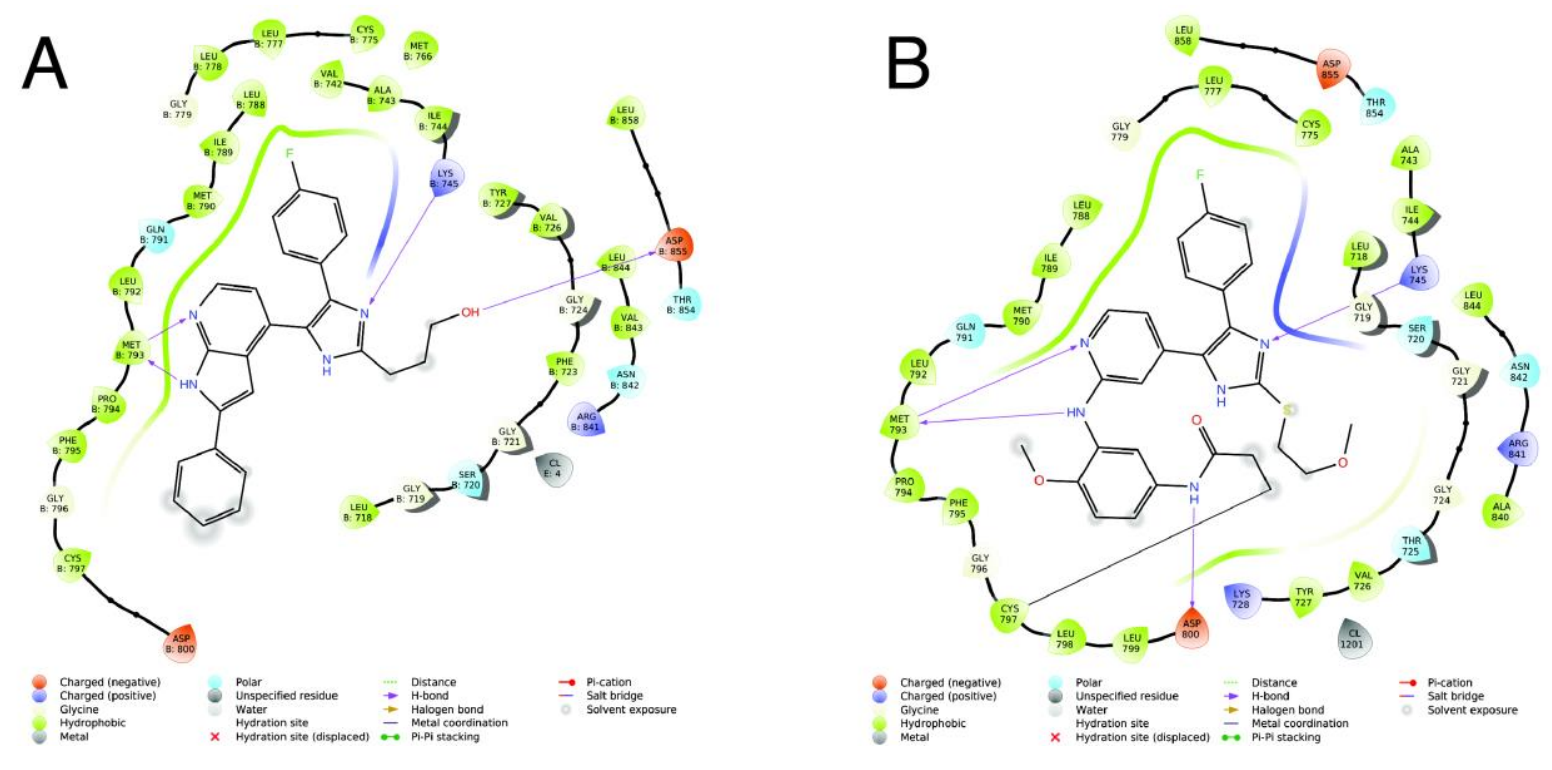

Figure S1. Representative LigPlot diagrams of 1 (A) and 4 (B) in complex with EGFR(T790M/V948R). 


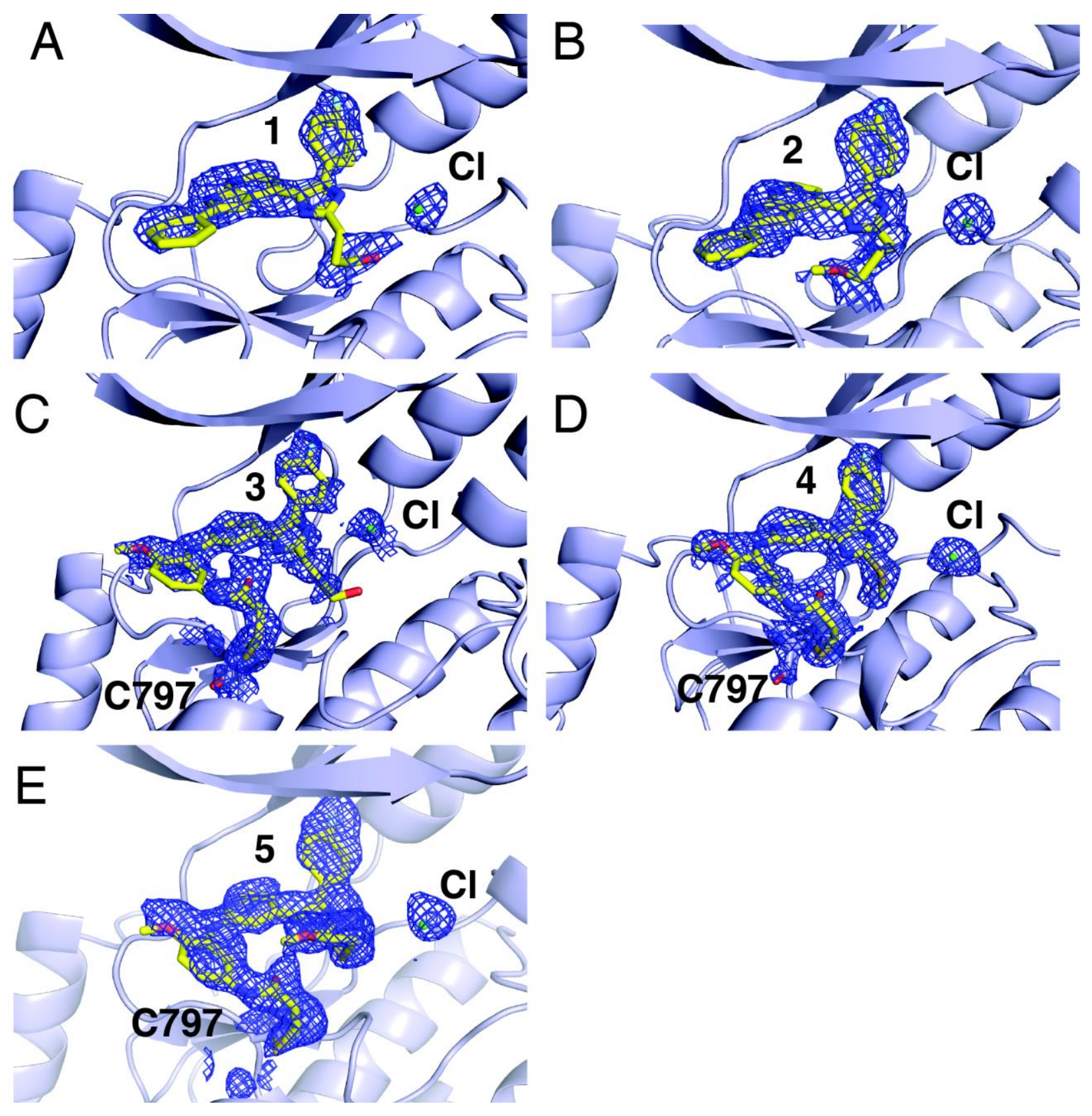

Figure S2. mFo-Fc omit maps for EGFR(T790M/V948R) crystal structures with ligand and chloride ion generated by PHENIX for A) 1 B) 2 C) 3 D) 4 E) 5 (contour level $1.5 \sigma$ ) 


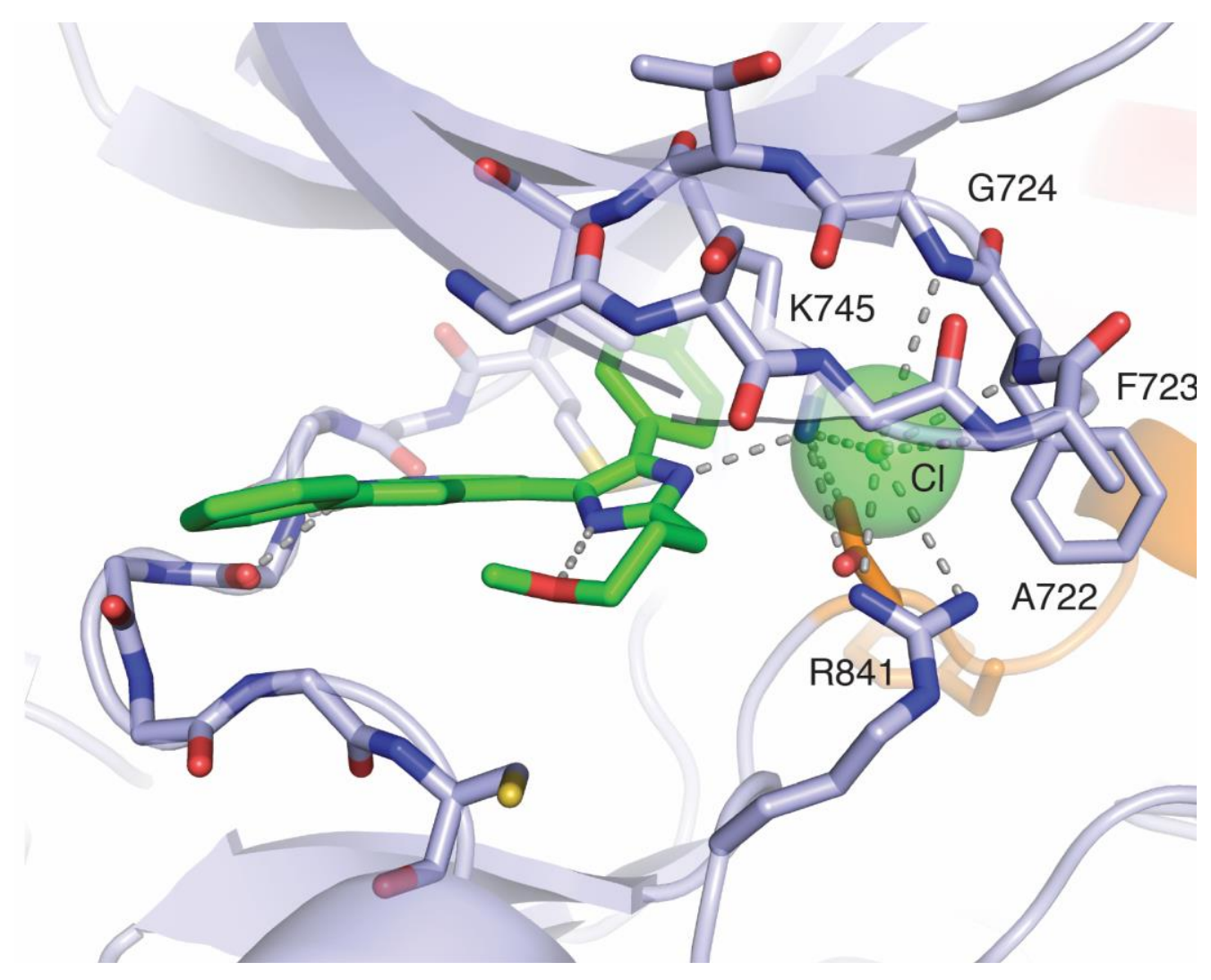

Figure S3. Extended view of Figure 2B illustrating the interactions with the ion (modeled as chloride) and positive side and main chain atoms of the EGFR(T790M/V948R) kinase domain. 
Table S2. Crystallographic data collection and refinement statistics for WT EGFR crystals.

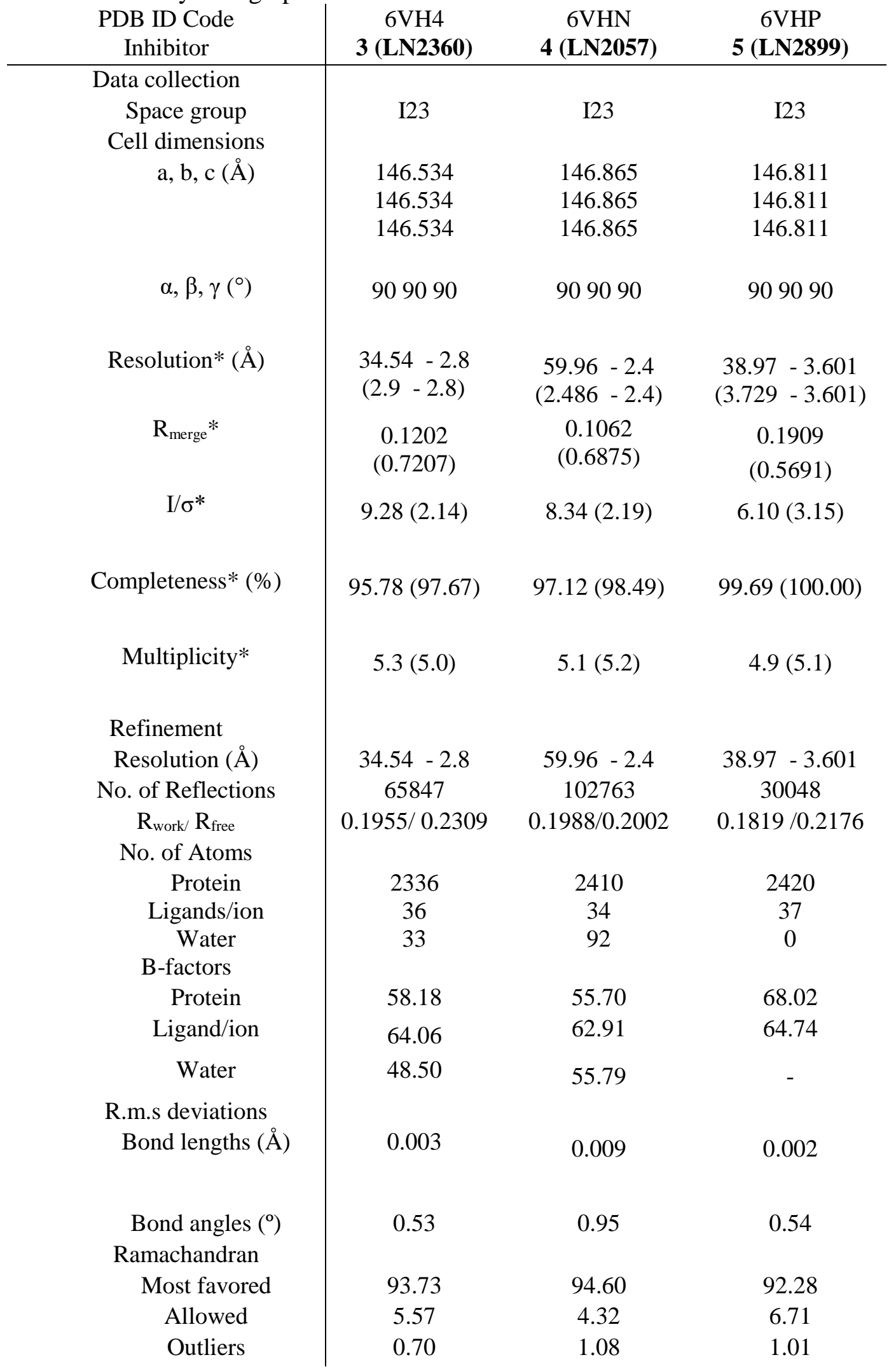

*Numbers in parentheses are for the highest resolution shell. 

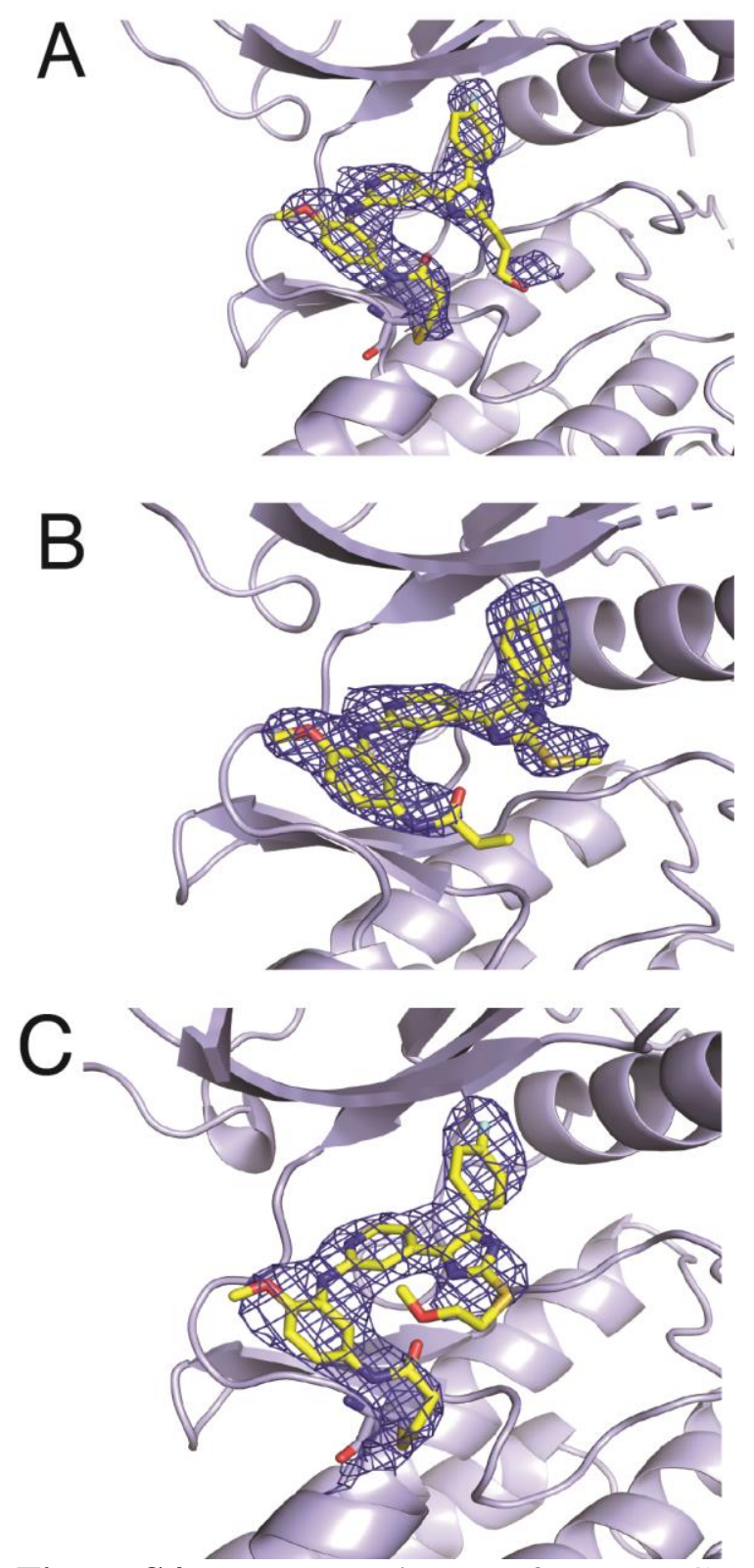

Figure S4. mFo-Fc omit maps for WT EGFR crystal structures with ligand and chloride ion generated by PHENIX for A) 3 B) 4 C) 5 (contour level $1.5 \sigma$ ). 
Table S3. Crystallographic data collection and refinement statistics of intermediate $\mathbf{2 6}$

\begin{tabular}{|l|l|}
\hline Space group & $\mathrm{P}-1$ (triclinic) \\
\hline Cell dimensions & determinate from 10562 reflections with $2.6^{\circ}<\theta<28.4^{\circ}$ \\
\hline a,b,c $(\AA)$ & $8.1901(7), 8.9234(8), 9.1834(9)$ \\
\hline$\beta\left({ }^{\circ}\right)$ & $92.006(7)$ \\
\hline $\mathrm{V}\left(\AA^{3}\right), \mathrm{z}$ & $568.54(10), 2$ \\
\hline Crystal size $\left(\mathrm{mm}^{3}\right)$ & $0.11 \times 0.28 \times 0.50($ colorless block) \\
\hline Range of Measurement & $2^{\circ} \leq \theta \leq 28^{\circ}-10 \leq \mathrm{h} \leq 10-11 \leq \mathrm{k} \leq 11-11 \leq 1 \leq 12$ \\
\hline No. of reflections: & \\
\hline Measured & 4968 \\
\hline Unique & $2691\left(\mathrm{R}_{\text {int }}=0.0468\right)$ \\
\hline Observed $(\mid \mathrm{F} / \sigma(\mathrm{F})>4.0)$ & $2348(\mid \mathrm{F} / \sigma(\mathrm{F})>4.0)$ \\
\hline Refinement & \\
\hline Nr. of parameters & 147 \\
\hline wR2 & 0.1368 \\
\hline R1 $($ observed), R(all) & $0.0506,0.0601$ \\
\hline Goodness of Fit & 1.063 \\
\hline Max. deviation of parameters & $0.001 *$ e.s.d \\
\hline Max. Peak final & \\
\hline diff. Fourier synthesis $(\mathrm{e} \AA-3)$ & $1.3,-1.92$ \\
\hline
\end{tabular}


Figure S5: X-ray structure of regioisomeric pure intermediate $\mathbf{2 6}$

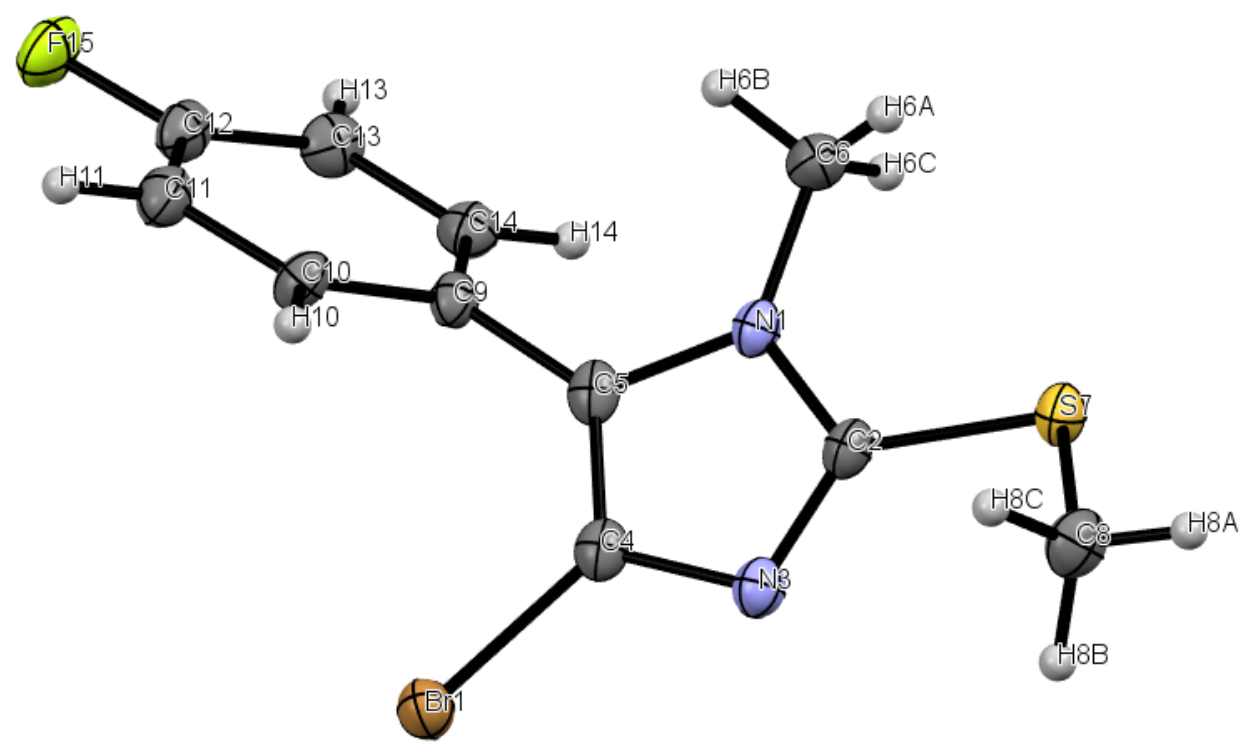

Table S4. Inhibitory activities of structurally characterized imidazole inhibitors not presented in Table 1.

\begin{tabular}{l|ll} 
compound \# & IC50 (EGFR-L858R/T790M) & IC50 (EGFR-L858R/T790M/C797S) \\
\hline $\mathbf{1}$ & $6.6 \mathrm{nM}$ & $21 \mathrm{nM}$ \\
$\mathbf{2}$ & $14 \mathrm{nM}$ & $6 \mathrm{nM}$ \\
$\mathbf{3}$ & $<0.5 \mathrm{nM}$ & $8 \mathrm{nM}$ \\
$\mathbf{5}$ & $1 \mathrm{nM}$ & $35 \mathrm{nM}$
\end{tabular}




\section{$\mathrm{N}$-(3-Bromo-4-methoxyphenyl)acrylamide:}

$1.00 \mathrm{~g} \mathrm{(4.95} \mathrm{mmol)} \mathrm{3-Bromo-4-methoxyaniline} \mathrm{was} \mathrm{dissolved} \mathrm{in} 10 \mathrm{ml}$ dry THF and $10 \mathrm{ml}$ of an aqueous $1 \mathrm{M} \mathrm{NaHCO}_{3}$ solution was added slowly. After cooling the biphasic mixture down to 0 ${ }^{\circ} \mathrm{C}, 420 \mu \mathrm{l}(5.20 \mathrm{mmol})$ acryloyl chloride was added dropwise under vigorous stirring. After complete addition the reaction mixture was warmed to room temperature and quenched by the addition of a saturated aqueous $\mathrm{NH}_{4} \mathrm{Cl}$ solution. The aqueous phase was extracted three times with DCM. The combined organic layers were dried over $\mathrm{Na}_{2} \mathrm{SO}_{4}$, filtered and the volatiles evaporated under reduced pressure. The crude product was triturated with DCM to give the title compound in $80 \%$ yield $(1.01 \mathrm{~g}, 3.96 \mathrm{mmol}) .1 \mathrm{H} \mathrm{NMR}(200 \mathrm{MHz}, \mathrm{DMSO}) \delta 10.13(\mathrm{~s}, 1 \mathrm{H}), 8.01$ $(\mathrm{d}, \mathrm{J}=2.4 \mathrm{~Hz}, 1 \mathrm{H}), 7.55(\mathrm{dd}, \mathrm{J}=8.9,2.5 \mathrm{~Hz}, 1 \mathrm{H}), 7.09(\mathrm{~d}, \mathrm{~J}=9.0 \mathrm{~Hz}, 1 \mathrm{H}), 6.47-6.16(\mathrm{~m}, 2 \mathrm{H})$, $5.81-5.68(\mathrm{~m}, 1 \mathrm{H}), 3.81(\mathrm{~s}, 3 \mathrm{H}) .13 \mathrm{C}$ NMR (50 MHz, DMSO) $\delta 162.94,151.57,133.06$, 131.64, 126.87, 123.84, 119.81, 112.77, 110.12, 56.30. ESI-MS: 256.1/257.1 [M+H] $]^{+}$. 